\title{
Transmittance measurement of a heliostat facility used in the preflight radiometric calibration of Earth-observing sensors
}

\author{
J. Czapla-Myers ${ }^{* a}$, K. Thome ${ }^{b}$, N. Anderson ${ }^{a}$, J. McCorkel ${ }^{a}$, N. Leisso ${ }^{a}$, W. Good ${ }^{c}$, and S. Collins ${ }^{c}$ \\ "Remote Sensing Group, College of Optical Sciences, University of Arizona, Tucson, Arizona, USA \\ DASA Goddard Space Flight Center, Greenbelt, Maryland, USA \\ ${ }^{c}$ Ball Aerospace and Technologies Corporation, Boulder, Colorado, USA
}

\begin{abstract}
Ball Aerospace and Technologies Corporation in Boulder, Colorado, has developed a heliostat facility that will be used to determine the preflight radiometric calibration of Earth-observing sensors that operate in the solar-reflective regime. While automatically tracking the Sun, the heliostat directs the solar beam inside a thermal vacuum chamber, where the sensor under test resides. The main advantage to using the Sun as the illumination source for preflight radiometric calibration is because it will also be the source of illumination when the sensor is in flight. This minimizes errors in the pre- and post-launch calibration due to spectral mismatches. It also allows the instrument under test to operate at irradiance values similar to those on orbit.

The Remote Sensing Group at the University of Arizona measured the transmittance of the heliostat facility using three methods, the first of which is a relative measurement made using a hyperspectral portable spectroradiometer and well-calibrated reference panel. The second method is also a relative measurement, and uses a 12-channel automated solar radiometer. The final method is an absolute measurement using a hyperspectral spectroradiometer and reference panel combination, where the spectroradiometer is calibrated on site using a solar-radiation-based calibration.
\end{abstract}

Keywords: heliostat, radiometric calibration, transmittance

\section{INTRODUCTION}

The preflight calibration of space borne sensors that operate in the solar reflective region is typically completed in the laboratory using a spherical integrating source that is illuminated by tungsten lamps. The radiance at the exit port of the sphere is varied by changing the number of lamps used to illuminate the sphere, and can be monitored with the use of a transfer radiometer. ${ }^{1}$ One concern with the use of lamps as a preflight radiometric calibration source for Earth-observing sensors is the spectral mismatch between the two sources. The operating temperature of tungsten lamps is on the order of $3000 \mathrm{~K}$, while the Sun can be approximated as a $6000-\mathrm{K}$ blackbody. In addition, the solar spectrum contains Fraunhofer lines that are not present in laboratory sources. Such differences may lead to higher uncertainty in sensors with narrow spectral bandwidths. ${ }^{2}$ It is advantageous to perform the preflight calibration using the same source that will be used on orbit, and one method that has been successfully used is the solar-radiationbased calibration (SRBC), where the instrument views a well-calibrated reference panel that is illuminated by the Sun. ${ }^{3,4}$ One disadvantage to such an approach is that the instrument is removed from the controlled laboratory environment.

One possible solution is to build a preflight calibration procedure that uses the Sun as a source while retaining a safe, controlled laboratory environment. Ball Aerospace and Technologies Corporation (BATC) has developed a heliostat to transfer sunlight into a thermal vacuum (TV) chamber where a sensor under test can run in operational conditions. Test criteria were established in order to evaluate the heliostat performance, and they include the spectral transmission, polarization sensitivity, and beam uniformity. The Remote Sensing Group (RSG) traveled to Boulder as part of the heliostat evaluation in November, 2008. The work presented here describes the methodology and results of the tests used to determine the spectral transmittance of the heliostat.

* j.czapla-myers(doptics.arizona.edu; phone 15206214242 ; fax 15206218292 ; www.optics.arizona.edu/rsg 


\section{HELIOSTAT DESCRIPTION AND OPERATION}

The heliostat facility developed at BATC in Boulder, Colorado, has been operational since late $2008 .{ }^{5}$ It is designed to work in the solar-reflective spectral region $(430-2300 \mathrm{~nm})$, and has the capability to operate year round. It uses three mirrors to guide the solar beam into a $3-\mathrm{m}$ diameter TV chamber, where a sensor undergoing preflight calibration can reside in a controllable, stable environment that is free from contamination. A $66-\mathrm{cm}$ diameter mirror on the roof of the heliostat building (M3) uses a solar ephemeris program to track the Sun throughout the day during operation. M3 directs the beam to a second $66-\mathrm{cm}$ diameter mirror (M2) that also resides on the roof. $\mathrm{M} 2$ directs the beam from the roof down to the floor where it is reflected from a $61-\mathrm{cm}$ diameter mirror (M1) into the TV chamber (Figure 1). The mirror diameters are chosen so that the beam diameter is approximately $33 \mathrm{~cm}$ at the TV chamber window, which is $35.5 \mathrm{~cm}$ in diameter. Each mirror is constructed of glass similar to BK7 and is coated with enhanced aluminum, which was chosen for its thermal characteristics and durability. The transmittance of the complete heliostat-atmospheric system is required to be greater than 0.20 for the spectral region ranging from $430-2300 \mathrm{~nm}$, not including absorption bands. Modeling of the mirrors by BATC has indicated that the total reflectance should be greater than 0.75 in the $400-2300-\mathrm{nm}$ spectral range for an angle of incidence of $45^{\circ} .5$

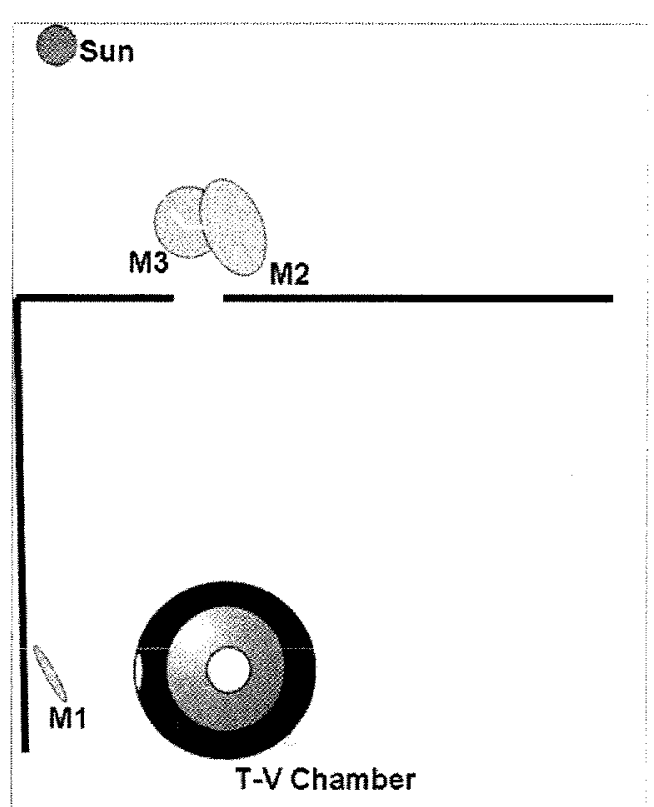

Figure 1: Heliostat layout, showing the tracking mirror (M3) and the other two relay mirrors (M2 and M1).

\section{TRANSMITTANCE MEASUREMENTS}

\subsection{Measurement philosophy}

RSG's original plan to measure the heliostat transmittance was based on the use of two transfer radiometers that were developed at the University of Arizona. ${ }^{1,6}$ The characterization process was to be determined using two pathways. The first relied on measurements made of a diffuser panel illuminated by the Sun, with and without the heliostat in the path. The second pathway was to act as a check of the method, and relied on measurements of atmospheric transmittance with a solar radiometer and that of the solar beam via the heliostat. Unfortunately, the use of transfer radiometers was deemed unsuitable due to the lack of space between the TV chamber window and the third heliostat mirror (M1) in the optical path.

The philosophy of the measurements presented in this work remains the same but the instrumentation is changed to accommodate the limited space between the TV chamber window and M1. Three techniques are used to measure the spectral transmittance of the heliostat:

- Relative radiance mode

- Solar radiometer mode (also a relative measurement)

- Absolute radiance mode

\subsection{Relative radiance mode}

The relative radiance mode uses the radiance reflected from a reference panel both inside and outside the heliostat to determine the transmittance. The strategy is to have an Analytical Spectral Devices (ASD) hyperspectral spectroradiometer view a reference panel inside the heliostat at the TV chamber that is illuminated by the heliostat beam, and also outside in the direct solar path using the same illumination and viewing geometry.

Prior to the measurements of the heliostat beam, the BATC reference panel and ASD are moved outside so that the direct solar beam, which acts as the reference, can be measured. The panel is mounted normal to the Sun and the ASD foreoptic is placed in its mount, which is the same as inside the heliostat. Data are taken with the panel 
unshaded and then shaded, thereby allowing the diffuse component of solar irradiance to be removed leaving only the direct-solar component.

The ASD and reference panel are quickly moved inside, and the heliostat measurement sequence begins with an ASD coupled to an $8^{\circ}$ FOV foreoptic viewing the BATC reference panel at a $45^{\circ}$ angle of incidence while it is mounted in front of the TV chamber window normal to the solar beam. The radiance reflected from the panel contains energy from sources other than the direct solar illumination, and therefore must be characterized. Blocking the solar beam incident on the first mirror (M3) gives the radiance from skylight and all sources along the path between M3 to the reference panel. Blocking M2 gives the radiance from all sources along the path from M2 to the reference panel. These sources include lights inside the test building. Finally, blocking M1 determines the radiance that is present between M1 and the reference panel which is also due to sources that are inside the building. The primary measurement is that which is made while blocking $\mathrm{M} 3$, since this characterizes all non-direct-solar radiance sources. Other parasol measurements are used to assess the scattered light effects of the heliostat system. To summarize, the measurement sequence inside the heliostat is as follows:

- Heliostat illuminated by global solar irradiance (no shading)

- M3 shaded with parasol

- No shading

- M2 shaded with parasol

- No shading

- M1 shaded with parasol

- No shading

The temporal atmospheric conditions are monitored throughout the measurement sequence using an automated solar radiometer (ASR) on the roof of the heliostat facility.

The transmittance of the heliostat, $\mathrm{T}_{\text {helio }}$, is determined using

$$
T_{\text {hello }}=\frac{V_{\text {in }}-V_{\text {diffuse (in) }}}{V_{\text {out }}-V_{\text {diffuse (out) }}} \frac{\tau_{a(\text { out })}}{\tau_{a(\text { in })}}
$$

where $V_{\text {in }}$ is the output of the ASR when measuring the panel inside the heliostat, $V_{\text {diffuse (in) }}$ is the output when measuring the panel with $\mathrm{M} 3$ blocked, $V_{\text {out }}$ is the output when the panel is outside and illuminated by direct and diffuse solar irradiance, $V_{\text {diffuse (out) }}$ is the output when the panel is shaded by a parasol, $\tau_{a \text { (out) }}$ is the atmospheric transmission when the reference panel is outside, and $\tau_{\mathrm{a}(\mathrm{in})}$ is the atmospheric transmission when the reference panel is in the heliostat. The atmospheric transmittance terms on the right of equation (1) create a correction factor that takes into account any changing atmospheric conditions between the two panel measurements.

\subsection{Solar radiometer mode}

The second relative approach employs a pair of ASRs to measure the direct solar irradiance before and after the heliostat. The instruments chosen for this work are ASR 33 and ASR 2K. ASR 33 is the newest radiometer produced by RSG, and it has twelve channels in the VNIR and SWIR regions of the spectrum. The narrow-bandwidth channels are centered at $380,400,411,550,670$, $780,870,940,1030,1250$, and $1550 \mathrm{~nm}$. The ASR 2K is a tenchannel radiometer that has the same ten VNIR bands $(380-$ $1030 \mathrm{~nm}$ ) as the ASR 33, but it has been retrofitted with two SWIR channels for this work (Figure 2). ${ }^{7}$ The FOV of the SWIR channels of both ASRs are designed to mimic the FOV of the ten VNIR channels, and they are mounted so that optical axis is also in line with the ten VNIR channels.

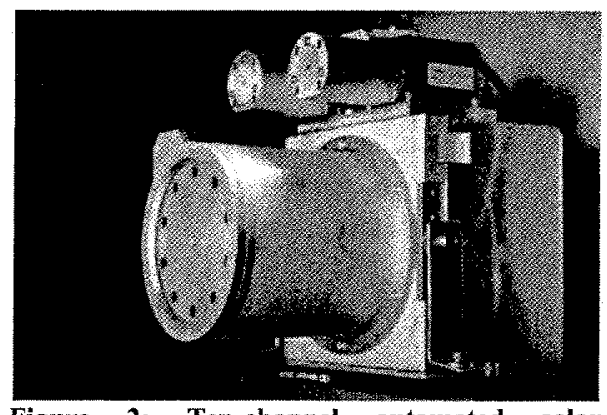

Figure 2: Ten-channel automated solar radiometer (serial number $2 \mathrm{~K}$ ) with two SWIR channels mounted on top of main unit. 
The measurements begin with the both ASRs operating on the roof of the heliostat building. The ASR 33 operates in normal automated mode and tracks the Sun throughout the process. The ASR $2 \mathrm{~K}$ operates in normal automated mode for several minutes in order to obtain a reference measurement, and then the head is removed from the tracking base and brought inside the building. It is placed in a mount between the last mirror in the optical path (MI) and the TV chamber where it measures the solar irradiance that passes through the heliostat (Figure 3). During the measurements sequence, a parasol is placed in front of $\mathrm{M} 3$ to block the direct solar beam so that the diffuse component can be measured. In addition, M2 and M1 are blocked sequentially so that any stray light present inside the building can be measured.

The basic determination for the heliostat transmittance, $T_{\text {helio, }}$ for each of the 12 spectral bands is given by

$$
T_{\text {helio }}=\frac{V_{2 K(\text { in })}}{V_{2 K(\text { out })}}
$$

where $V_{2 K}$ (in) is the output voltage from ASR $2 \mathrm{~K}$ while operating inside the heliostat, and $V_{2 K}$ (out) is the output voltage of ASR $2 \mathrm{~K}$ while operating on the roof. The data are uncorrected at this point, since the atmospheric transmission may change from the time of the rooftop measurements to when ASR $2 \mathrm{~K}$ is placed inside the heliostat. The data from ASR 33 are used to correct for any changes in atmospheric transmission, and equation (2) becomes

$$
T_{\text {helio }}=\frac{V_{2 K(\text { in })}}{V_{2 K(\text { out })}} \frac{V_{33(t 1)}}{V_{33(t 2)}}
$$

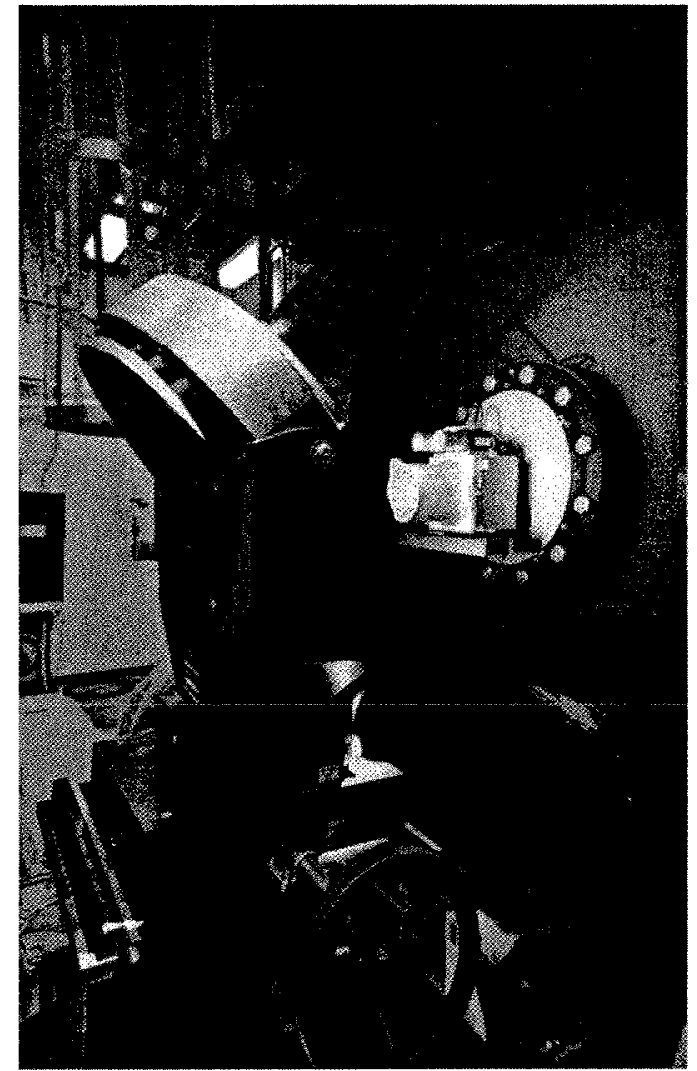

Figure 3: 12-channel ASR 2K measuring the solar irradiance at the thermal vacuum chamber window. M1 is to the left of the ASR.

where $V_{33(t 1)}$ is the output voltage of ASR 33 taken when ASR $2 \mathrm{~K}$ is still on the roof, and $V_{33 \text { (t2) }}$ is the output voltage when ASR $2 \mathrm{~K}$ is in the heliostat.

\subsection{Absolute radiance mode}

The heliostat transmittance is also determined by measuring the radiance from a $46-\mathrm{cm}$ Spectralon panel that is placed at the TV chamber window location and illuminated while normal to the heliostat beam. A calibrated ASD spectroradiometer with $1^{\circ}$ full FOV foreoptic views the panel at a $45^{\circ}$ angle of incidence. During these measurements, an ASR is operating continuously on the roof to determine the temporal atmospheric transmittance. The barometric pressure collected by RSG's all-sky monitor package is used to infer the molecular scattering at arbitrary wavelengths. Analysis of the spectral transmittance in the 12 spectral bands of ASR 33 provides the aerosol characterization that permits inference of the aerosol scattering transmittance at arbitrary wavelengths. Ancillary data from suitable web sources provide column ozone amounts that are used to determine the ozone transmittance. The atmospheric transmittance is combined with the Thullier (2002) solar model to determine the solar irradiance at M3.

The radiance measured by the ASD while viewing the panel divided by the panel BRDF gives the incident irradiance on the panel. The ratio of the incident irradiance on the panel to that on M3 gives the transmittance of the heliostat system.

The radiance from the panel included energy from sources that were not the direct solar illumination. Blocking the solar beam onto M3 gives the radiance from skylight and all sources along the path between M3 and the panel. In 
addition to blocking M3, other measurements were made with M2 and M1 covered by the parasol. Blocking M2 gives the radiance from all sources along the path from $\mathrm{M} 2$ to the Spectralon panel, and blocking M1 gives the radiance from all sources along the path from M1 to the Spectralon panel.

The transmission of the heliostat, $\mathrm{T}_{\text {helio }}$, is given by

$$
T_{\text {helio }}=\frac{E_{p}}{E_{M 3}}
$$

where $E_{p}$ is the irradiance on the panel after the last mirror in the heliostat, and $E_{M 3}$ is the irradiance at mirror M3 at the beginning of the heliostat. The spectral irradiance at the panel, $\mathrm{E}_{\mathrm{p}}$, is given by

$$
E_{p}=\frac{\pi}{\rho_{p}} L_{p} \quad\left[W m^{-2} \mu m^{-1}\right]
$$

where $\rho_{p}$ is the bidirectional reflectance factor (BRF) of the panel, and $L_{p}$ is the spectral radiance reflected from the panel. The spectral irradiance at M3 on the roof is given by

$$
E_{M 3}=E_{0} \tau_{a} \quad\left[W m^{-2} \mu m^{-1}\right]
$$

where $E_{0}$ is the spectral exoatmospheric irradiance, and $\tau_{\mathrm{a}}$ is the spectral atmospheric transmission. Combining equations (5) and (6), the transmission of the heliostat becomes

$$
T_{\text {helio }}=\frac{\pi L_{p}}{\rho_{p} E_{0} \tau_{a}}
$$

The radiance reflected from the reference panel, $L_{p}$, is measured using an ASD that has been absolutely calibrated using the solar-radiation-based calibration technique. The reference panel BRF is measured in RSG's laboratory, the exoatmospheric irradiance used in this work is that of Thullier (2002), and the atmospheric transmission is determined using data from RSG's 12-channel solar radiometer.

\subsection{Uncertainty}

Prior to the on-site heliostat measurements in Boulder, the level of uncertainty that one would expect when using the Sun as a source for preflight calibration was evaluated. ${ }^{8}$ The uncertainty of the relative radiance mode measurements originate from three sources: 1) the changing atmospheric transmission during the reference and heliostat measurements, 2) the repeatability of the panel being mounted normal to the solar beam and the ASD view angle to the panel, and, 3) the ability to block out all non-direct-solar sources of illumination. A properly-designed parasol is capable of blocking the direct solar beam so that the diffuse sky irradiance can be determined, so this term is considered negligible. While outside, the panel is mounted normal to the Sun using a pinhole alignment system, and the ASD foreoptic is attached to the panel mount in a v-block where the view angle is repeatable to within $\pm 1^{\circ}$. These two sources of uncertainty are also considered to be negligible. The uncertainty in the relative measurements is therefore driven primarily by the uncertainty in atmospheric transmittance, which is determined to be less than $0.5 \%$ in the wavelength range of the ASR $(380-1030 \mathrm{~nm})$.

The transmittance of the heliostat using solar radiometer mode does not require the use of a reference panel. The ASRs are viewing the direct solar beam, so there are no uncertainties introduced because of panel alignment issues. There is also no uncertainty introduced due to panel BRF issues, so the measurement of atmospheric transmission is the primary uncertainty source. As in the case of the relative radiance mode, it is estimated to be less than $0.5 \%$.

The uncertainty in the absolute radiance measurement is determined by the uncertainty in the solar exoatmospheric irradiance, the panel BRF, the calibration of the ASD, and the atmospheric transmittance. Previous work has shown that the SRBC method has a worst-case (e.g. blue region of the spectrum) uncertainty of approximately $3.5 \% .{ }^{1}$ When the uncertainty is coupled to the others, one can expect an overall uncertainty of approximately $3.7 \%$ in the blue region, and this value would decrease with increasing wavelength. 


\section{RESULTS}

\subsection{Relative radiance mode}

The results of the three-mirror heliostat transmittance measured using an ASR and reference panel in relative mode are shown in Figure 4. The two spectral regions near 1400 and $1900 \mathrm{~nm}$ are not shown due to increased noise caused by low signal-to-noise ratio (SNR). The shape of the hyperspectral transmittance curve has the same shape that is expected by simply cubing the reflectance of the three mirrors.

The correction of the heliostat transmittance due to changing atmospheric conditions between the reference and transmittance measurement is shown in Figure 5. At $350 \mathrm{~nm}$, the transmittance correction is approximately $2 \%$, and it decreases until approximately $560 \mathrm{~nm}$, where there is no correction factor applied to the data.

\subsection{Solar radiometer mode}

The heliostat transmittance measured using the solar radiometer mode is shown in Figure 6 , where the data from the relative radiance mode are shown for comparison. The percent difference between the twelve ASR channels and the hyperspectral ASR measurements at each ASR wavelength is shown in Table 1. The difference is generally less than $2.5 \%$ from $380 \mathrm{~nm}$ to $1000 \mathrm{~nm}$, and above $1000 \mathrm{~nm}$ the difference is on the order of $1 \%$ or less.

\subsection{Absolute radiance mode}

The results for the heliostat transmittance as measured using the absolute radiance mode are shown in Figure 7, which also shows the hyperspectral relative measurements from Section 4.1 for comparison. The percent difference between the two methods is shown in Figure 8 , where it is shown as a 20 -nm running average to increase clarity. The two methods show similar results in the VNIR region of the spectrum, but the absolute measurements become noisier out past $1000 \mathrm{~nm}$. This is due to a combination of spectral mismatches between the ASD and the Thullier solar model, absorption features (e.g. the 760-nm O $\mathrm{O}_{2}$ band), and low SNR in the ASD.

\subsection{Atmosphere-heliostat transmission}

The requirement set forth by BATC is that on an atmospherically-clear day, the three-mirror heliostat, the atmosphere, and the TV chamber

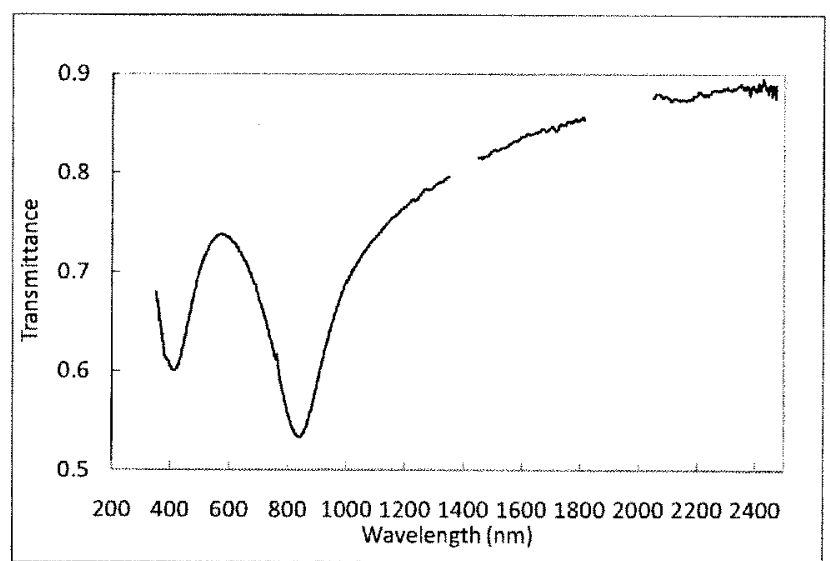

Figure 4: Heliostat transmittance as measured using the relative radiance mode with an $\mathrm{ASD}$ portable spectroradiometer and reference panel.

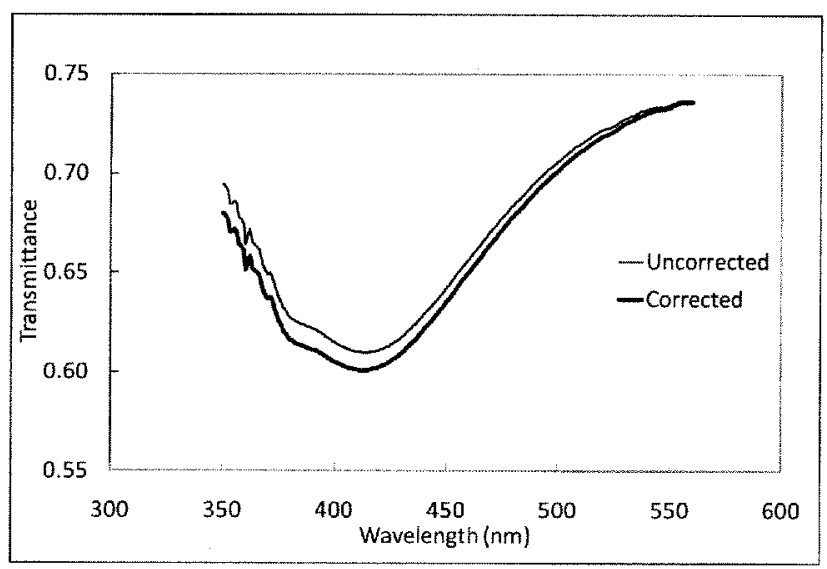

Figure 5: The heliostat transmittance before and after a correction factor is applied using the reference solar radiometer on the roof.

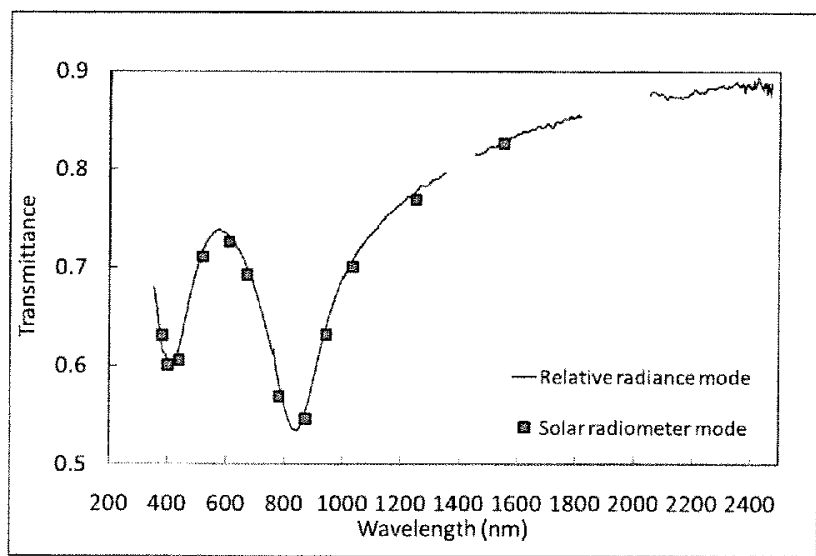

Figure 6: Heliostat transmittance measured using the solar radiometer mode, which is also a relative measurement. 
window will have a transmittance greater than 0.20 . The hyperspectral heliostat transmittance data from Section 4.1 are multiplied by the hyperspectral atmospheric transmittance determined using an ASR and ancillary atmospheric data, and the results are shown in Figure 9 for a solar zenith angle (SZA) of $77.6^{\circ}$. Operating the heliostat at a smaller SZA (or higher solar elevation) for a given set of atmospheric conditions reduces the path length through the atmosphere, thereby increasing the transmission. Figure 9 also shows the theoretical transmission through the atmosphere and heliostat when the solar elevation is higher $\left(30^{\circ}\right.$ and $\left.60^{\circ}\right)$ for similar atmospheric conditions.

\section{CONCLUSIONS}

RSG was present at BATC's facility in November 2008 to measure the transmittance of a newlydeveloped heliostat that will be used to perform the preflight calibration of Earth-observing sensors. The results of this work show that the three-mirror heliostat has a transmittance greater than 0.5 throughout the $430-2300-\mathrm{nm}$ spectral range. The specified transmittance of the atmosphere, heliostat, and TV chamber window is to be greater than 0.2 . Unfortunately, the TV chamber window could not be measured as part of the heliostat system because of logistical issues, but it is anticipated that the window can be coated such that the transmittance of the entire system does not decrease to below the 0.2 specification in the given wavelength range.

The results of this work show that two relative methods, which rely on different instrumentation, agree to within approximately $\pm 2.5 \%$. The largest differences occur at wavelengths below $1000 \mathrm{~nm}$, and between 1000 and $1550 \mathrm{~nm}$, the percent difference is on the order of $1 \%$.

Overall, the absolute measurements are in good agreement with the relative measurements. The SRBC of the ASD completed in Boulder was in the late afternoon when the Sun was at a large solar zenith angle. The preferential time for SRBCs is generally in the morning when the atmospheric stability is greater. The increased uncertainty in the atmospheric transmission translates to an increase in the uncertainty in the absolute calibration of the ASD.
Table 1: Percent difference in heliostat transmittance as measured using relative radiance (ASD) and solar radiometer (ASR) mode.

\begin{tabular}{cc}
$\begin{array}{c}\text { Wavelength } \\
\text { (nm) }\end{array}$ & $\begin{array}{c}\text { Percent Difference } \\
\text { [(ASR-ASD)/ASR] }\end{array}$ \\
\hline 380 & 2.3 \\
400 & -0.8 \\
439 & -2.5 \\
520 & -1.1 \\
610 & -0.7 \\
670 & -0.9 \\
781 & -2.0 \\
872 & -1.7 \\
943 & -1.7 \\
1033 & -1.1 \\
1250 & -1.1 \\
1550 & 0.0
\end{tabular}

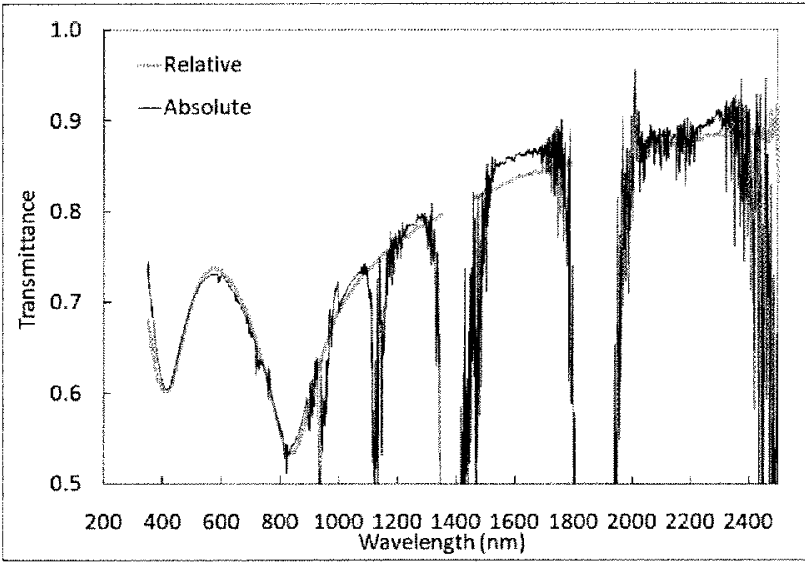

Figure 7: Heliostat transmittance of three-mirror system measured using absolute mode, and compared to relative mode.

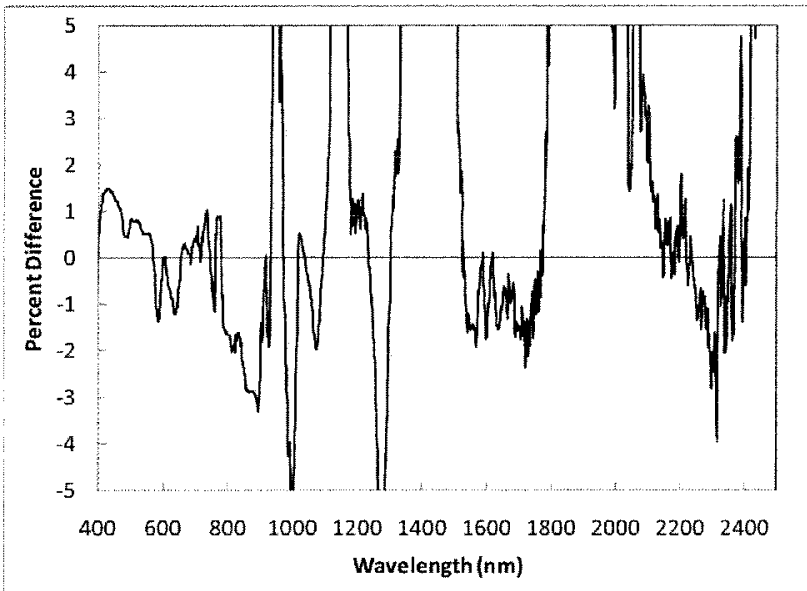

Figure 8: Percent difference in the relative and absolute measurements of heliostat transmittance. The results are shown as a 20-nm running average to increase visual clarity. 


\section{ACKNOWLEDGEMENTS}

This work is supported by Ball Aerospace and Technologies Corporation contract number 228493.

\section{REFERENCES}

[1] S. F. Biggar, "Calibration of a visible and near-infrared portable transfer radiometer," Metrologia, 35, 701-706 (1998).

[2] D. E. Flittner, and P. N. Slater, "Stability of Narrow-Band Filter Radiometers in the Solar-Reflective Range," Photogrammetric Engineering and Remote Sensing, 57(2), 165-171 (1991).

[3] R. A. Barnes, R. E. Eplee Jr., S. F. Biggar, K. J. Thome, E. F. Zalewski, P. N. Slater, and A. W. Holmes, [The SeaWiFS Solar-Radiation-Based

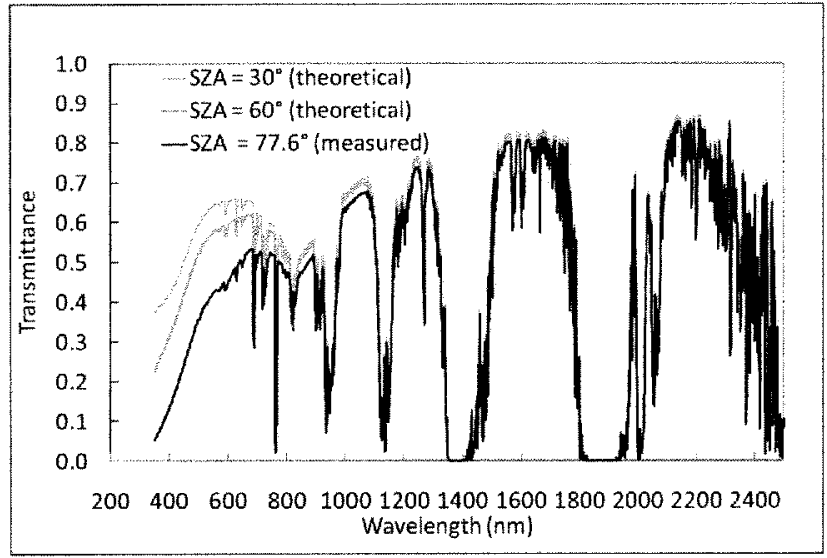

Figure 9: Hyperspectral transmittance of the BATC heliostat system, including a clear atmosphere and the three-mirror heliostat. The solar zenith angle (SZA) during these measurements is $77.6^{\circ}$. The transmittance for the same atmosphere-heliostat combination is shown for a theoretical $30^{\circ}$ and $60^{\circ} \mathrm{SZA}$.

Calibration and the Transfer-to-Orbit Experiment] NASA, (1999).

[4] S. F. Biggar, P. N. Slater, K. J. Thome, A. W. Holmes, and R. A. Barnes, "Preflight Solar-based calibration of SeaWiFS." SPIE 1939, 233-242 (1993).

[5] M. A. Kuester, J. Czapla-Myers, P. Kaptchen, W. Good, T. Lin, R. To, S. Biggar, and K. Thome, "Development of a heliostat facility for solar-radiation-based calibration of earth observing sensors." SPIE 7081, 708119-1 - 708119-8 (2008).

[6] P. R. Spyak, D. S. Smith, J. Thiry, and C. Burkhart, "Short-wave infrared transfer radiometer for the calibration of the Moderate-Resolution Imaging Spectrometer and Advanced Spaceborne Thermal Emission and Reflection Radiometer," Applied Optics, 39(31), 5694-5706 (2000).

[7] A. R. Ehsani, J. A. Reagan, and W. H. Erxleben, "Design and Performance Analysis of an Automated 10Channel Solar Radiometer Instrument," Journal of Atmospheric and Oceanic Technology, 15(3), 697-707 (1998).

[8] K. J. Thome, J. S. Czapla-Myers, and M. A. Kuester, "Accuracy assessment for the radiometric calibration of Earth-observing imagers using preflight techniques relying on the sun as a source," SPIE, 7081, 7081181 - $708118-12$ (2008). 